Research Article

\title{
Central Extension of Left Alternative Algebras and the Second Cohomology Group
}

\author{
Said Boulmane ii \\ Department of Economic Sciences and Management, Moulay Ismail University, Errachidia, Morocco \\ Correspondence should be addressed to Said Boulmane; said.boulmane@gmail.com
}

Received 21 April 2021; Revised 13 July 2021; Accepted 19 July 2021; Published 28 July 2021

Academic Editor: Sergejs Solovjovs

Copyright (c) 2021 Said Boulmane. This is an open access article distributed under the Creative Commons Attribution License, which permits unrestricted use, distribution, and reproduction in any medium, provided the original work is properly cited.

The purpose of this paper is to prove that the second cohomology group $H^{2}(A, \mathbb{F})$ of a left alternative algebra $A$ over an algebraically closed field $\mathbb{F}$ of characteristic 0 can be interpreted as the set of equivalent classes of one-dimensional central extensions of $A$.

\section{Introduction}

In this paper, we consider finite-dimensional alternative algebras over an algebraically closed field $\mathbb{F}$ of characteristic 0 . An algebra $A$ is left alternative (resp. right alternative) if it satisfies the left alternative law (resp. the right alternative law) $x^{2} y=x(x y)$ (resp. $\left.y x^{2}=(y x) x\right)$, for all $x, y \in A$. An alternative algebra is one which is both left and right alternative. For a general theory about alternative algebras, refer $[1-6]$.

If $A$ is left alternative algebra, then the algebra defined on the same vector space $A$ with "opposite" multiplication $x \diamond y:=y x$ is a right alternative algebra and vice versa. Hence, all the statements for left alternative algebras have their corresponding statements for right alternative algebras. Thus, we will only consider the left alternative algebra case in this paper.

Elhamdadi and Makhlouf [7] introduced an algebraic cohomology of left alternative algebras, and they computed the second cohomology group of the 2 by 2 matrix algebra $\mathscr{M}_{2}(\mathbb{F})$ (considered as left alternative algebra). In this paper, we recall the cohomology theory and birepresentations for left alternative algebra and we discuss the links between the second cohomology group $H^{2}(A, \mathbb{F})$ and central extensions of left alternative algebra, and then we prove that the second cohomology group $H^{2}(A, \mathbb{F})$ of a left alternative algebra $A$ can be interpreted as the set of classes of one-dimensional central extensions of $A$.

\section{Preliminaries}

In this section, we recall some definitions and concepts of alternative algebras. This class of algebras is well studied by several authors; for more details, refer to $[1,4,6]$.

Definition 1. Let $A$ be a left alternative algebra. A birepresentation of $A$ is a triple $(M, \pi, \Pi)$, where $M$ is a $\mathbb{F}$-vector space and $\pi: A \longrightarrow \operatorname{End}(M)$ and $\Pi: A \longrightarrow \operatorname{End}(M)$ are two linear maps satisfying

(i) $\pi\left(a^{2}\right)=(\pi(a))^{2}$

(ii) $\Pi\left(a a^{\prime}\right)-\Pi\left(a^{\prime}\right) \Pi(a)=\left[\Pi\left(a^{\prime}\right), \pi(a)\right]$

where

$$
\left[\Pi\left(a^{\prime}\right), \pi(a)\right]=\Pi\left(a^{\prime}\right) \pi(a)-\pi(a) \Pi\left(a^{\prime}\right), \quad \forall a, a^{\prime} \in A .
$$

In this case, $M$ is called bimodule of $A$. We denote $\pi(a) m$ (resp. $\Pi(a) m)$ by a.m (resp. m.a). 
Proposition 1. Let $A$ be a left alternative algebra and $M$ be a bimodule of $A$. The direct summand $A \oplus M$ is made into a left alternative algebra, by defining multiplication as follows:

$$
(a+m)\left(a^{\prime}+m^{\prime}\right)=a a^{\prime}+a \cdot m^{\prime}+m \cdot a^{\prime},
$$

for all $a, a^{\prime} \in A$ and $m, m^{\prime} \in M$.

Proof. We show that the left alternative law is satisfied. Let $a, a^{\prime} \in A$ and $m, m^{\prime} \in M$.

$$
\begin{aligned}
(a+m)^{2}\left(a^{\prime}+m^{\prime}\right)-(a+m)\left[(a+m)\left(a^{\prime}+m^{\prime}\right)\right]= & \left(a^{2}+m \cdot a+a \cdot m\right)\left(a^{\prime}+m^{\prime}\right)-(a+m)\left(a a^{\prime}+m \cdot a^{\prime}+a \cdot m^{\prime}\right) \\
= & a^{2} a^{\prime}+a^{2} \cdot m^{\prime}+(m \cdot a) \cdot a^{\prime}+(a \cdot m) \cdot a^{\prime}-a\left(a a^{\prime}\right)-a \cdot\left(m \cdot a^{\prime}\right) \\
& -a \cdot\left(a \cdot m^{\prime}\right)-m \cdot\left(a a^{\prime}\right) \\
= & a^{2} \cdot m^{\prime}+(m \cdot a) \cdot a^{\prime}+(a \cdot m) \cdot a^{\prime}-a \cdot\left(m \cdot a^{\prime}\right)-a \cdot\left(a \cdot m^{\prime}\right)-m \cdot\left(a a^{\prime}\right) \\
= & (m \cdot a) \cdot a^{\prime}+(a \cdot m) \cdot a^{\prime}-a \cdot\left(m \cdot a^{\prime}\right)-m \cdot\left(a a^{\prime}\right) \\
= & 0 .
\end{aligned}
$$

Therefore, $A \oplus M$ is a left alternative algebra.

\section{Central Extensions of Left Alternative Algebras}

Definition 2. Let $A$ be a left alternative algebra; a one-dimensional central extension of $A$ is an exact sequence of left alternative algebras:

$$
0 \longrightarrow \mathbb{F} \stackrel{i}{\longrightarrow} \tilde{A} \stackrel{\pi}{\longrightarrow} A \longrightarrow 0
$$

such that $\operatorname{Ker}(\pi) \subset \boldsymbol{Z}(\widetilde{A})$.

We denote by $\mathcal{Z}(\widetilde{A})=\{a \in \widetilde{A}, \widetilde{A} a=0$ and $a \widetilde{A}=0\}$ the center of $\widetilde{A}$ and by $\operatorname{Ker}(\pi)$ the Kernel of $\pi$.

Two such extensions $\widetilde{A}$ and $\widetilde{A}^{\prime}$ are called equivalent if there is a morphism $\phi: \widetilde{A} \longrightarrow \widetilde{A}^{\prime}$ of left alternative algebras such that the following diagram is commutative:

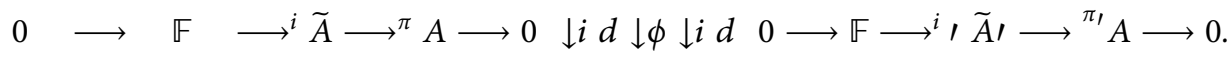

Denote by $C L(A, \mathbb{F})$ the set of equivalent classes of onedimensional central extensions of $A$.

Example 1. Let $A_{1}$ be a left alternative algebra, $V$ a vector space, and $\varphi: A_{1} \times A_{1} \longrightarrow V$ be a bilinear map such that

$$
\varphi\left(a^{2}, b\right)=\varphi(a, a b), \quad \text { for all } a, b \in A_{1} .
$$

We define the following product on the vector space direct sum $A=A_{1} \oplus V$ :

$$
(a+v)\left(b+v^{\prime}\right):=a b+\varphi(a, b), \quad \text { for all } a, b \in A_{1}, v, v^{\prime} \in V .
$$

We can check easily that $A$ is a left alternative algebra (see Example 1 in [8]), and further we obtain an exact sequence:

$$
0 \longrightarrow V \stackrel{\lambda}{\longrightarrow} A_{1} \oplus V \stackrel{\mu}{\longrightarrow} A_{1} \longrightarrow 0 .
$$

Moreover, we have $\operatorname{Ker} \mu=\{0\} \times V \subset \mathbb{Z}\left(A_{1} \oplus V\right)$, so the extension is central and called the central extension of $A_{1}$ by $V$ via $\varphi$.

\section{Interpretation of $H^{2}(A, \mathbb{F})$}

Now, we recall the cohomology theory for left alternative algebra introduced by M. Elhamdadi and A. Makhlouf [7].
Let $A$ be a left alternative algebra and $M$ an $A$-bimodule. If $p \geq 1$, a $p$-cochain of $A$ with values in $M$ are a $p$-linear mapping of $A^{p}$ in $M$. We denote by $C^{p}(A, M)$ the space of the $p$-cochains of $A$. For $p<0$, we put $C^{p}(A, M)=\{0\}$ and $C^{0}(A, M)=M$.

For $\varphi \in C^{1}(A, M)$, we define the first differential $\delta^{1} \varphi \in C^{2}(A, M)$ by

$$
\delta^{1} \varphi(a, b)=\varphi(a) \cdot b+a \cdot \varphi(b)-\varphi(a b), \quad \text { for all } a, b \in A .
$$

Let $\varphi \in C^{2}(A, M)$, we define the second differential $\delta^{2} \varphi \in C^{3}(A, M)$ by

$$
\delta^{2} \varphi=\delta_{H}^{2} \varphi^{\circ}\left(i d^{3}+\sigma_{1}\right),
$$

where $\sigma_{1}$ is the trilinear map on $A^{3}$ defined by $\sigma_{1}(a, b, c)=$ $(b, a, c)$ and $\delta_{H}^{2}$ denotes the Hochschild differential of degree 2 defined by

$$
\delta_{H}^{2} \varphi(a, b, c)=a \cdot \varphi(b, c)-\varphi(a b, c)+\varphi(a, b c)-\varphi(a, b) . c .
$$

Call any $p$-form $\varphi \in C^{p}(A, M)$ a $p$-cocycle if and only if $\delta^{p} \varphi=0$ and denote the subspace of $p$-cocycles by $Z^{p}(A, M)$.

The $p$-th cohomology group $H^{p}(A, M)$ is defined to be the factor space: 


$$
\frac{Z^{p}(A, M)}{\delta^{p-1} C^{p-1}(A, M)}, \quad \text { for } p \geq 1
$$

Remark 1. In Example 1, if we consider $V$ as an $A_{1}$-trivial bimodule, then

$$
\delta_{H}^{2} \varphi(a, b, c)=\varphi(a, b c)-\varphi(a b, c) .
$$

It follows that

$$
\begin{aligned}
\delta^{2} \varphi(a, b, c) & =\delta_{H}^{2} \varphi(a+b, a+b, 2 c), \\
& =\varphi(a+b,(a+b)(2 c))-\varphi((a+b)(a+b), 2 c) \\
& =0 .
\end{aligned}
$$

Consequently, $A_{1} \oplus V$ is a left alternative algebra if and only if $\varphi \in Z^{2}\left(A_{1}, V\right)$, (i.e., $\varphi$ is a 2-cocycle).

Now, from our previous discussion, we obtain the main result of this paper:

Proposition 2. Let $A$ be a left alternative algebra and consider $\mathbb{F}$ as a trivial A-bimodule, then the cohomology group $H^{2}(A, \mathbb{F})$ can be interpreted as the set of equivalent classes of one-dimensional central extensions of $A$ (i.e., there is a bijection between $H^{2}(A, \mathbb{F})$ and $C L(A, \mathbb{F})$ ).

Proof. Let $\varphi \in Z^{2}(A, \mathbb{F})$, we can associate to $\varphi$ the extension

$$
0 \longrightarrow \mathbb{F} \stackrel{i}{\longrightarrow} A \oplus \mathbb{F} \stackrel{\pi}{\longrightarrow} A \longrightarrow 0,
$$

where the product of $A \oplus \mathbb{F}$ is given by $(a, \alpha)(b, \beta)=(a b, \varphi(a, b))$, for all $a, b \in A$ and $\alpha, \beta \in \mathbb{F}$. If $\bar{\varphi}=\bar{\varphi}^{\prime}$, then $\varphi^{\prime}=\varphi-\delta^{1} \theta$, with $\theta \in C^{1}(A, \mathbb{F})$. Moreover, $\varphi^{\prime}$ defines a one-dimensional central extension:

$$
0 \longrightarrow \mathbb{F} \stackrel{i}{\longrightarrow} A \oplus \mathbb{F} \stackrel{\pi}{\longrightarrow} A \longrightarrow 0,
$$

where the product of $A \oplus \mathbb{F}$ is given by

$$
(a, \alpha)(b, \beta)=(a b, \varphi \prime(a, b))=\left(a b, \varphi(a, b)-\delta^{1} \theta(a, b)\right) .
$$

The mapping $\phi: A \oplus_{\varphi} \mathbb{F} \longrightarrow A \oplus_{\varphi_{1}} \mathbb{F} \quad$ defined by $(a, \alpha) \longrightarrow(a, \alpha+\theta(a))$ is a morphism of left alternative algebras. Indeed, let $a, b \in A$ and $\alpha, \beta \in \mathbb{F}$.

$$
\begin{aligned}
\phi((a, \alpha)(b, \beta))= & \phi(a b, \varphi(a, b)), \\
= & (a b, \varphi(a, b)+\theta(a b)) \\
= & \left(a b, \varphi(a, b)-\delta^{1} \theta(a, b)\right), \\
& \left(\text { Since } \delta^{1} \theta(a, b)=-\theta(a b)\right) \\
= & \left(a b, \varphi^{\prime}(a, b)\right) \\
= & (a, \alpha+\theta(a))(b, \beta+\theta(b)) \\
= & \phi(a, \alpha) \phi(b, \beta),
\end{aligned}
$$

which gives the equivalent between these extensions. So, cohomologous cocycles correspond to equivalent one- dimensional central extensions. We also prove that equivalent one-dimensional central extensions induce cohomologous cocycles. In order to show the subjectivity, we have to construct a 2-cocycle from a given one-dimensional central extension of $A$ :

$$
0 \longrightarrow \mathbb{F} \stackrel{i}{\longrightarrow} \tilde{A} \stackrel{\pi}{\longrightarrow} A \longrightarrow 0 .
$$

This is given as follows:

There is a linear map $\beta: A \longrightarrow \widetilde{A}$ with $\pi \circ \beta=i d_{A}$. Let $\varphi(a, b)=\beta(a) \beta(b)-\beta(a b)$, for $a, b \in A$. As $\pi$ is a morphism of left alternative algebras, we have $\pi(\varphi(a, b))=0$; thus, $\varphi(a, b)$ has values in $\operatorname{ker}(\pi)=i m(i)=\mathbb{F}$. Then, one can check that the map $\varphi: A \times A \longrightarrow \mathbb{F}$ is a 2-cocycle. We thus obtain

$$
H^{2}(A, \mathbb{F}) \cong C L(A, \mathbb{F})
$$

\section{Data Availability}

No data were used to support this study.

\section{Conflicts of Interest}

The author declares that there are no conflicts of interest.

\section{References}

[1] N. Jacobson, "Structure of alternative and Jordan bimodules," Osaka Journal of Mathematics, vol. 6, pp. 1-71, 1954.

[2] K. McCrimmon, "Alternative algebras," 2021, http://mysite. science.uottawa.ca/neher/Papers/alternative/.

[3] N. I. Sandu, "Free Moufang loops and alternative algebras," Buletinul Academiei de Ştiinţe a Republicii Moldova Matematica, vol. 132, no. 2, pp. 313-316, 2009.

[4] R. D. Schafer, An Introduction to Nonassociative Algebras, Academic Press, New York, NY, USA, 1996.

[5] I. P. Shestakov, "Moufang loops and alternative algebras," Proceedings of the American Mathematical Society, vol. 132, pp. 313-316, 2004.

[6] K. A. Zhevlakov, A. M. Slin'ko, I. P. Shestakov, and A. I. Shirshov, "Rings that are nearly associative," Pure and Applied Mathematics, Vol. 104, Academic Press, Inc, New York, NY, USA, 1982.

[7] M. Elhamdadi and A. Makhlouf, "Cohomology and formal deformations of alternative algebras," Journal of Generalized Lie Theory and Applications, vol. 5, p. 10, Article ID G110105, 2011.

[8] M. Ait Ben Haddou and S. Boulmane, "Pseudo-euclidean alternative algebras," Communications in Algebra, vol. 44, no. 12, pp. 5199-5222, 2016. 